\title{
Pulsars as the Sources of High Energy Cosmic Ray Positrons
}

\author{
Dan Hooper \\ Theoretical Astrophysics, Fermi National Accelerator Laboratory, Batavia, USA and \\ Department of Astronomy and Astrophysics, The University of Chicago, USA \\ Pasquale Blasi \\ Theoretical Astrophysics, Fermi National Accelerator Laboratory, Batavia, USA \\ INAF-Osservatorio Astrofisico di Arcetri, Firenze, Italy and \\ INFN-Laboratori Nazionali del Gran Sasso, Assergi, L'Aquila, Italy \\ Pasquale Dario Serpico \\ Physics Department, Theory Division, CERN, CH-1211 Geneva 23, Switzerland and \\ Theoretical Astrophysics, Fermi National Accelerator Laboratory, Batavia, USA
}

\begin{abstract}
Recent results from the PAMELA satellite indicate the presence of a large flux of positrons (relative to electrons) in the cosmic ray spectrum between approximately 10 and $100 \mathrm{GeV}$. As annihilating dark matter particles in many models are predicted to contribute to the cosmic ray positron spectrum in this energy range, a great deal of interest has resulted from this observation. Here, we consider pulsars (rapidly spinning, magnetized neutron stars) as an alternative source of this signal. After calculating the contribution to the cosmic ray positron and electron spectra from pulsars, we find that the spectrum observed by PAMELA could plausibly originate from such sources. In particular, a significant contribution is expected from the sum of all mature pulsars throughout the Milky Way, as well as from the most nearby mature pulsars (such as Geminga and B0656+14). The signal from nearby pulsars is expected to generate a small but significant dipole anisotropy in the cosmic ray electron spectrum, potentially providing a method by which the Fermi gamma-ray space telescope would be capable of discriminating between the pulsar and dark matter origins of the observed high energy positrons.
\end{abstract}

PACS numbers: 98.70.Sa;97.60.Gb;95.35.+d;98.70.Sa FERMILAB-PUB-08-429-A, CERN-PH-TH/2008-228

\section{INTRODUCTION}

Dark matter particles annihilating in the Galactic Halo are predicted to generate a number of potentially observable products, including gamma-rays, electrons, positrons, protons and antiprotons. In contrast to gamma-rays, which travel along straight lines, charged particles move under the influence of the Galactic Magnetic Field, diffusing and losing energy, resulting in a diffuse spectrum at Earth. By studying the cosmic ray antimatter spectra, balloon or satellite-based experiments hope to identify signatures of dark matter.

The PAMELA satellite, which began its three-year mission in June of 2006, is designed to measure the spectra of cosmic ray positrons up to $270 \mathrm{GeV}$ and electrons up to $2 \mathrm{TeV}$, each with unprecedented precision [1]. Recent results show that the ratio of positrons to electrons plus positrons (the positron fraction) in the cosmic ray spectrum appears to stop decreasing and begins to climb quite rapidly between $10 \mathrm{GeV}$ and $100 \mathrm{GeV}$ 2]. A similar trend was in fact also indicated by earlier experiments, including HEAT [3] and AMS-01 [4], although with lesser statistical significance.

The behavior in the positron fraction observed by PAMELA is very different from that predicted for secondary positrons produced in the collisions of cosmic ray nuclides with the interstellar medium. Barring systematics (e.g. problems in the positron/proton separation at high rigidity), the data appear to indicate the existence of additional, primary sources of high energy positrons, such as dark matter annihilations taking place in the halo of the Milky Way [5, 6, 7, 8, 9, 10]. It should be noted, however, that while the observed spectral shape can be easily accommodated with annihilating dark matter, the normalization of this contribution to the PAMELA data requires a somewhat large annihilation rate. Such a large rate could, in principle, result from the dark matter possessing an annihilation cross section in excess of the value predicted for a simple $s$-wave thermal relic $\left(\sigma v \sim 3 \times 10^{-26} \mathrm{~cm}^{3} / \mathrm{sec}\right)$, for example due to the Sommerfeld effect [6, 10, 11] or in the case that the WIMP is of non-thermal origin [12]. Alternatively, a large annihilation rate could be generated as the result of significant inhomogeneities in the spatial distribution of dark matter, such as the presence of a large, nearby dark matter subhalo [13]. In either case, such scenarios are somewhat constrained by observations of gamma-rays [14, antiprotons [9, 12] and synchrotron emission 12, 15].

The challenges involved in explaining the PAMELA signal with annihilating dark matter lead us to consider a less exotic and purely astrophysical explanation for the observed positron flux. Energetic electron-positron pairs can be in fact produced in astrophysical sources, the leading candidate sites being pulsars - rapidly spinning, magnetized neutron stars, which emit pulsed electromagnetic radiation, as observed from Earth. 
In this paper, we explore the possibility that the positron fraction reported by PAMELA may be generated by mature pulsars. Gamma-ray pulsars are predicted to produce energetic electron-positron pairs with a harder spectrum than that from secondary cosmic-ray induced origin, leading to the possibility that such sources may dominate the cosmic ray positron spectrum at high energies. We calculate the spectrum of such particles from known local pulsars (Geminga and B0656+14), and from the sum of all pulsars distributed throughout the Milky Way. As found in earlier studies [16], we find that both local pulsars and the sum of pulsars distributed throughout the Milky Way can contribute significantly to the observed spectrum. At $10 \mathrm{GeV}$, we estimate that on average only $\sim 20 \%$ of the cosmic ray positrons originate from pulsars within 500 parsecs from the Solar System. If gamma-ray pulsars are formed at a rate of $\sim 4$ per century in the Milky Way, we find that the observed flux of $\sim 10-20 \mathrm{GeV}$ positrons could be plausibly generated in such objects. Similar conclusions were derived in [17, 18]. Above $\sim 50 \mathrm{GeV}$, however, the positron spectrum is likely to be dominated by a single or small number of nearby pulsars. If the high energy electron-positron spectrum is dominated by a single nearby source, it opens the possibility of detecting a dipole anisotropy in their angular distribution (see also [19]). We find that such a feature could potentially be detected by the Fermi gamma-ray space telescope (formerly known as GLAST) [20], thus enabling a powerful test to discriminate between the pulsar and dark matter origins of the observed cosmic ray positron excess.

The remainder of this article is structured as follows: In Sec. II we review the known properties of pulsars and consider them as sources of high energy electron-positron pairs. In Sec. III, we consider the nearby pulsars Geminga and B0656+14 and discuss their potential contributions to the cosmic ray positron spectrum. In Sec. [V] we calculate the expected dipole anisotropy from nearby pulsars and compare this to the sensitivity of the Fermi gamma-ray space telescope. We summarize and draw our conclusions in Sec. V

\section{PULSARS AS SOURCES OF ELECTRON-POSITRON PAIRS}

In both models of polar gap [21, 22] and outer gap [23], electrons can be accelerated in different regions of the pulsar magnetosphere and induce an electromagnetic cascade through the emission of curvature radiation, which in turn results in production of photons which are above threshold for pair production in the strong pulsar magnetic field. This process results in lower energy electrons and positrons that can escape the magnetosphere either through the open field lines [25] or after joining the pulsar wind [18]. In this second case, the electrons and positrons lose part of their energy adiabatically because of the expansion of the wind. The energy spectrum injected by a single pulsar depends on the environmental parameters of the pulsar, but some attempts to calculate the average spectrum injected by a population of mature pulsars suggest that the spectrum may be relatively hard, having a slope of 1.5-1.6 [18]. This spectrum, however, results from a complex interplay of individual pulsar spectra, of the spatial and age distributions of pulsars in the Galaxy, and on the assumption that the chief channel for pulsar spin down is magnetic dipole radiation. Due to the related uncertainties, variations from this injection spectra cannot be ruled out. Typically, one concentrates the attention on pulsars of age $\sim 10^{5}$ years because younger pulsars are likely to still be surrounded by their nebulae, which confine electrons and positrons and thus prevent them from being liberated into the interstellar medium until later times.

Still, some energetics considerations can be done with simple analytical models; this will also help the understanding of arguments developed in the next Section. The rate of energy injection from a single pulsar in the form of pairs is limited by its spin-down power (the rate of energy loss corresponding to the slowing rate of rotation). Assuming that this is simply due to the emission of magnetic dipole radiation, the maximum rate of energy injection can be written as (see e.g. [24]):

$$
\dot{E}=-\frac{B_{s}^{2} R_{s}^{6} \Omega^{4}}{6 c^{3}} \approx 10^{31} B_{12}^{2} R_{10}^{6} P^{-4} \operatorname{erg~s}^{-1},
$$

where $B_{12}=B_{s} / 10^{12} \mathrm{G}$ is the magnetic field at the surface of the star, $R_{10}=R_{s} / 10 \mathrm{~km}$ is the radius of the star and $P$ is the period of the star in seconds. The period $P$ (gyration frequency $\Omega$ ) increases (decreases) with time as a result of the spin-down, according to

$$
\Omega(t)=\frac{\Omega_{0}}{\left(1+t / \tau_{0}\right)^{1 / 2}},
$$

where $\tau_{0}=3 c^{3} I /\left(B_{s}^{2} R_{s}^{6} \Omega_{0}^{2}\right), I=(2 / 5) M_{s} R_{s}^{2}$ is the moment of inertia of the star with mass $M_{s}$ and $\Omega_{0}=2 \pi / P_{0}$ is the initial spin frequency of the pulsar and $P_{0}$ is the initial period. Numerically, this yields: 


$$
\tau_{0}=7.4 \times 10^{7} B_{12}^{-2}\left(\frac{M_{s}}{1.4 M_{\odot}}\right) R_{10}^{-4} P_{0}^{2} \text { years. }
$$

It follows that the upper limit to the rate of energy deposit in the form of electron-positron pairs is

$$
\mathcal{L}=I \Omega \dot{\Omega}=\frac{1}{2} I \Omega_{0}^{2} \frac{1}{\tau_{0}} \frac{1}{\left(1+\frac{t}{\tau_{0}}\right)^{2}}
$$

In terms of the total energy injected in a time $t$ after the pulsar birth,

$$
E_{t o t}(t)=\frac{1}{2} I \Omega_{0}^{2} \frac{t}{\tau_{0}} \frac{1}{1+\frac{t}{\tau_{0}}}=6 \times 10^{43} P_{0}^{-4} R_{10}^{6} B_{12}^{2} t_{5} \frac{1}{\left(1+\frac{t}{\tau_{0}}\right)} \mathrm{erg},
$$

where $t_{5}$ is the time in units of $10^{5}$ years. Therefore, the total energy that a mature pulsar $\left(t \gg \tau_{0}\right)$ has injected in the form of magnetic dipole radiation saturates to

$$
E_{t o t} \approx \frac{1}{2} I \Omega_{0}^{2}=2.2 \times 10^{46}\left(\frac{M_{s}}{1.4 M_{\odot}}\right) R_{10}^{2} P_{0}^{-2} \mathrm{erg} .
$$

In the same assumption of a mature pulsar, we also have that $\Omega_{0} \approx \Omega\left(t / \tau_{0}\right)^{1 / 2}$, where $\Omega$ is the gyration frequency measured today. For instance, for the Geminga pulsar $(P=230 \mathrm{~ms}) t \approx 370,000$ years and $\tau \approx 10^{4}$ years (using $B_{12}=1.6$ and $\left.R_{10}=1.5\right)$, one has $\Omega_{0} \approx 166 \mathrm{~s}^{-1}\left(P_{0} \approx 40 \mathrm{~ms}\right)$. For these values of the parameters, the total energy output of the pulsar is rather large, $E_{t o t} \approx 10^{49} \mathrm{erg}$, which could easily account for the high energy positron flux. It is worth stressing, however, that only a small fraction of this energy will eventually end up in the form of escaping electron-positron pairs, and thus this number should be treated as an absolute upper limit on the pair luminosity of a single pulsar. Qualitatively, the combined effect of a declining absolute luminosity [Eq. (44)] and of an increasing escape probability conspire in singling out typical ages of $\sim 10^{5}$ years for the pulsars expected to contribute maximally to the positron flux.

To proceed in a more quantitative way towards the calculation of the overall spectrum from Galactic pulsars, one needs to adopt a model for the $e^{+}-e^{-}$acceleration and escape probability from a single pulsar with a given magnetic field, period, etc. and then integrate over a Monte Carlo distribution of these typical parameters in a Galactic Pulsar population. The resulting injection spectrum we adopt follows from such a calculation in Ref. [18]:

$$
\frac{d N_{e}}{d E_{e}} \approx 8.6 \times 10^{38} \dot{N}_{100}\left(E_{e} / \mathrm{GeV}\right)^{-1.6} \exp \left(-E_{e} / 80 \mathrm{GeV}\right) \mathrm{GeV}^{-1} \mathrm{~s}^{-1}
$$

where $\dot{N}_{100}$ is the rate of pulsar formation in units of pulsars per century. This expression corresponds to an average energy output in electron-positron pairs of approximately $6 \times 10^{46} \mathrm{erg}$ per pulsar, i.e. to efficiency $\lesssim 1 \%$ compared with the upper bound derived above. In the following, we inject this spectrum according to the spatial distribution of pulsars given in Refs. [18, 26].

Once electrons and positrons are produced, diffusion in the Galactic Magnetic Field regulates their motion. Unlike previous approaches to the problem, mostly based on a simple implementation of the leaky box model, we calculate the effects of propagation by solving the transport equation for electrons, including synchrotron and inverse Compton scattering losses:

$$
\frac{\partial}{\partial t} \frac{d n_{e}}{d E_{e}}=\vec{\nabla} \cdot\left[K\left(E_{e}\right) \vec{\nabla} \frac{d n_{e}}{d E_{e}}\right]+\frac{\partial}{\partial E_{e}}\left[B\left(E_{e}\right) \frac{d n_{e}}{d E_{e}}\right]+Q\left(E_{e}, \vec{x}\right)
$$

with a free escape boundary condition at $4 \mathrm{kpc}$ above and below the Galactic Plane. Here $d n_{e} / d E_{e}$ is the number density of electrons/positrons per unit energy, $K\left(E_{e}\right)$ is the diffusion coefficient and $B\left(E_{e}\right)$ is the rate of energy loss. We adopt $K\left(E_{e}\right) \equiv K_{0}\left(1+E_{e} /(3 \mathrm{GeV})\right)^{\delta}$ with $K_{0}=3.4 \times 10^{28} \mathrm{~cm}^{2} / \mathrm{s}$ and $\delta=0.6$, and $B\left(E_{e}\right)=-b E_{e}^{2}$ with $b=10^{-16} \mathrm{GeV}^{-1} \mathrm{~s}^{-1}$. $Q$ corresponds to the source term described above.

In Fig. 1. we show the spectrum of positrons and the positron fraction resulting from the sum of all pulsars throughout the Milky Way. In the upper panels, we show results for different rates of pulsar birth (one per 10, 25, or 100 years). The dashed line represents the baseline result neglecting the contribution from pulsars, including only 

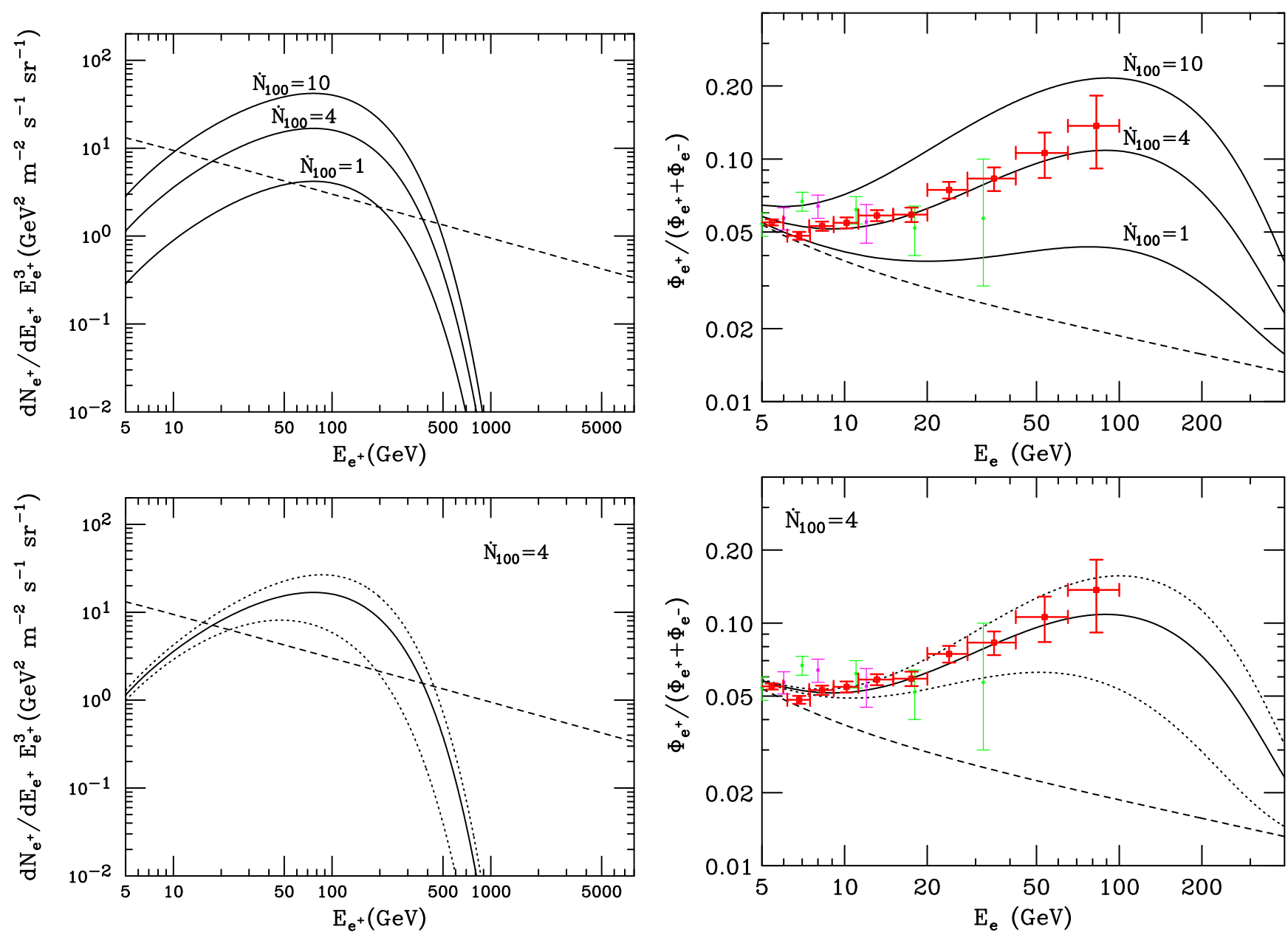

FIG. 1: The spectrum of cosmic ray positrons (left) and the positron fraction (right) resulting from the sum of all pulsars throughout the Milky Way. Also shown as a dashed line is the prediction for secondary positrons (and primary and secondary electrons in the right frames) as calculated in Ref. 27]. In the right frames, the measurements of HEAT [3] (light green and magenta) and measurements of PAMELA [2] (dark red) are also shown. We have used the injected spectrum reported in Eq. (77). In the lower frames, the upper (lower) dotted line represents the case in which the injection rate within 500 parsecs of the Solar System is doubled (neglected), providing an estimate the variance resulting from the small number of nearby pulsars contributing to the spectrum.

the positrons produced as secondaries in the hadronic interaction of cosmic rays. In the right frames, the positron ratio is obtained considering, besides secondary leptons, also the primary electrons accounted as in [27], to ease the comparison with previous literature. In the right frames, the measurements of HEAT [3] (light green and magenta) and the measurements of PAMELA [2] (dark red) are also shown.

In the lower frames of Fig. 1 we show the positron spectrum and the positron fraction for $\dot{N}_{100}=4$ if the injection rate within 500 parsecs of the Solar System is doubled (upper dotted curve) or neglected (lower dotted curve). This provides an estimate of the relative importance of average nearby sources compared to the contribution from more distant pulsars. We will discuss this issue further in Sec. III]

Interestingly, the best fit to the HEAT and PAMELA data appears to be obtained for $\dot{N}_{100}=4$, namely about one pulsar birth each $\sim 25$ years. It is worth noting that this number is only slightly higher than the typical estimates of the galactic core collapse supernovae rate, from which pulsars are formed. This rate has been estimated in a variety of ways, including from the scaling of rates in external galaxies, from the measured gamma-ray flux from galactic ${ }^{26} \mathrm{Al}$, from historical observations of galactic supernovae, and from empirical upper limits from neutrino observatories (for a review, see Ref. 28]). Also note that since the primary electron flux is determined from a fit to the absolute flux, which has uncertainties as large as $\pm 50 \%$ around $10 \mathrm{GeV}$ (see the cosmic ray review in Ref. 29]), the best-fit value of $\dot{N}_{100}$ extracted from the ratio is affected by at least an error as large. Additionally, in principle our numerical results could be modified if a different normalization for the diffusion coefficient were chosen; yet, the constraint on 
the confinement time inferred from measurements of the boron-to-carbon ratio does not leave much freedom in this respect, at least in the energy region of interest here. The slope of the diffusion coefficient in energy is also expected not to be a critical parameter, since the positron "excess" has thus far been detected over a relatively narrow region in energy, where $\delta$ should be virtually constant. Future observations will determine whether the actual excess positron spectrum extends to energies beyond $\sim 100 \mathrm{GeV}$.

The cutoff in the positron spectrum derived in our calculations is solely the result of the corresponding cutoff in the injection spectrum shown in Eq. (7). This cutoff is determined by the details of the development of the electromagnetic cascade in the pulsar magnetosphere and, even more importantly, by the distribution of periods, magnetic fields, and radii of mature pulsars. The exact value of the cutoff energy should, therefore, not be considered to be a robust prediction of the theory, although it represents a good estimate of the order of magnitude of the cutoff energy. For instance, in Ref. [25] it is argued that the typical energy of electrons and positrons in the cascade associated with the polar gap is $E_{e} \approx 8 B_{12}^{5 / 7} P_{0}^{-17 / 7} \mathrm{GeV}$ which, for a period of $200 \mathrm{~ms}$, would yield a maximum energy of the resulting pairs of $\sim 400 \mathrm{GeV}$. On the other hand, the description of Ref. [18] leads to different maximum energies ( $\sim 80 \mathrm{GeV})$ and even different scalings of the cutoff with the parameters of the pulsar. It is therefore not very difficult to accommodate moderately higher energy positrons in the pulsar scenario, if future observations were to show that this is the case. Having listed possible caveats to the above results, what is remarkable is that, without any fine-tuning of the models, typical predictions for both the spectrum and the normalization can explain the positron fraction data very well.

At energies lower than $\sim 10 \mathrm{GeV}$, pulsars are not expected to contribute any appreciable flux because of the very hard spectrum, compared to the spectrum of secondary positrons produced in hadronic interactions of cosmic rays diffusing throughout the Galaxy. These secondary electrons approximately reproduce the steep spectrum of the parent nuclei and at low energies dominate the observed positron fraction. Since the spectrum of positrons from pulsars is important only at relatively high energies, we have neglected here the role of solar modulation.

\section{NEARBY PULSARS AS A SOURCE OF HIGH ENERGY COSMIC RAY ELECTRONS AND POSITRONS}

In this section, following earlier studies [16, 19], we re-explore the possibility that an individual or small number of nearby pulsars dominate the cosmic ray positron spectrum within the energy range studied by PAMELA. As argued in the previous section, in order to contribute significantly such a pulsar can be neither too young nor too old. The electrons and positrons from very young pulsars (i.e. much younger than 100,000 yrs or $t_{5} \ll 1$ ) are expected to be confined by the surrounding pulsar wind nebula. Furthermore, electrons/positrons in the PAMELA energy range require $\sim 10^{4}(D / 100 \mathrm{pc})^{2}$ years to diffuse a distance, $D$. The electrons/positrons from very old pulsars, in contrast, will have diffused over a large volume, diluting their contribution to insignificant levels. There are at least two pulsars that appear to satisfy these constraints, Geminga which is estimated to be 157 parsecs from Earth and 370,000 years old, and B0656+14, which is estimated to be 290 parsecs from Earth and 110,000 years old.

The spectrum of electron positron pairs at Earth is again calculated by solving the transport equation, but this time for a single source. Moreover we consider the case of a bursting source, namely one in which the duration of the emission is much shorter than the travel time from the source. For the pulsars discussed above this seems indeed to be appropriate. For the simple case of a power-law spectrum of electrons and positrons, $Q\left(E_{e}\right) \propto E_{e}^{-\alpha}$, injected a time $t$ ago at a distance $D$ from the Solar System, the spectrum observed at Earth is given by:

$$
\frac{d N_{e}}{d E_{e}}=\frac{Q\left(E_{e}\right)}{\pi^{3 / 2} D^{3}}\left(1-b t E_{e}\right)^{\alpha-2}\left(\frac{D}{D_{\text {dif }}}\right)^{3} e^{-\left(D / D_{\text {dif }}\right)^{2}},
$$

where the diffusion distance scale is given by:

$$
D_{\mathrm{dif}}\left(E_{e}, t\right) \approx 2 \sqrt{K\left(E_{e}\right) t \frac{1-\left(1-E_{e} / E_{\max }\right)^{1-\delta}}{(1-\delta) E_{e} / E_{\max }}} .
$$

As a result of energy losses, the maximum energy that electrons can reach the observer with is approximately given by $E_{\max } \approx 1 /(b t) \approx 3 t_{5}^{-1} \mathrm{TeV}$. Since energy losses for electrons with energy $\sim 10-100 \mathrm{GeV}$ are not crucial over a time period of a few hundred thousand years, Eq. (10) approximately reduces to $D_{\text {dif }}\left(E_{e}, t\right) \sim \sqrt{4 K\left(E_{e}\right) t}$, corresponding to $\sim 300-500 \sqrt{t_{5}}$ parsecs over the energy range of 10 to $50 \mathrm{GeV}$.

As a first example, we consider the nearby $(D \simeq 157 \mathrm{pc})$ Geminga pulsar, having an estimated age of 370,000 years [16, 19]. In order to take into account the uncertainties in the injection spectrum, we carry out our calculations by assuming that electrons and positrons are injected with a spectral index $\alpha=2$ and 1.5, and exponentially cutoff 

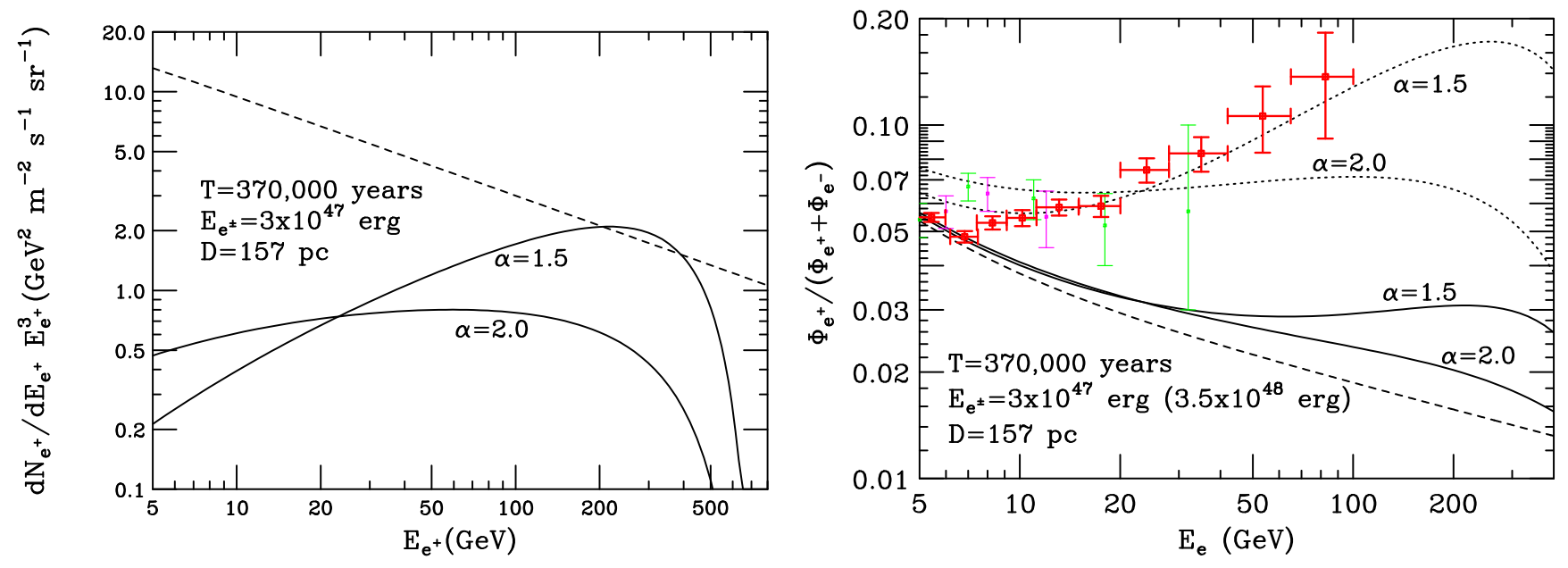

FIG. 2: The spectrum of positrons (left) and ratio of positrons to electrons plus positrons (right) from the pulsar Geminga, with the dashed lines as in Fig. 1. In the right frames, the measurements of HEAT [3] (light green and magenta) and measurements of PAMELA [2] (dark red) are also shown. Here we have used an injected spectrum such that $d N_{e} / d E_{e} \propto E^{-\alpha} \exp \left(-E_{e} / 600 \mathrm{GeV}\right)$, with $\alpha=1.5$ and 2.2. The solid lines correspond to an energy in pairs given by $3.5 \times 10^{47}$ erg, while the dotted lines require an output of $3 \times 10^{48} \mathrm{erg}$.

above $600 \mathrm{GeV}$. As a default quantity, we consider a total energy of $3 \times 10^{47}$ erg injected as electron-positron pairs, which constitutes a few percent of the total spin down power of the pulsar. Our results for Geminga are shown in Fig. 2. In the left panel we plot the positron spectrum from Geminga for $\alpha=1.5$ and 2. Again, the dashed line presents the spectrum from secondary positrons alone. The right panel shows the positron fraction for the two values of $\alpha$ and for two values of the total energy injected in pairs. The lower of these values in our default choice, $3 \times 10^{47}$ erg (solid lines), while the higher, dotted lines represent the approximate energy required to generate the entire flux of excess positrons from Geminga alone $\left(3.5 \times 10^{48} \mathrm{erg}\right)$. We thus conclude that if Geminga were to dominate the observed positron fraction at high energies, it would have to transfer on the order of $\sim 30 \%$ of its spin-down power into electron-positron pairs. Such a high efficiency to pairs appears unlikely. The (probably) subdominant role of Geminga is not particularly unexpected, given its relatively old age.

We now turn our attention to the case of the pulsar B0656+14. B0656+14 is considerably younger than Geminga (approximately 110,000 years old), has a period today of $P=390 \mathrm{~ms}$, and a current spin down luminosity that is approximately the same as Geminga. The spectrum of positrons and the positron fraction from B0656+14 are shown in the left and right panels of Fig. 3. respectively. The lines are labeled as in Fig. 2. Because of the younger age of this pulsar, the flux of positrons expected from B0656+14 is somewhat higher than from Geminga, despite being somewhat more distant $(D=290 \mathrm{pc})$. In the case with $\alpha=1.5$, the predicted positron fraction can fit the PAMELA data if it injected $\sim 8 \times 10^{47} \mathrm{erg}$ in electron-positron pairs. This appears still large, but less extreme than in the Geminga case.

In Sec. III we found that nearby pulsars ( $D \lesssim 500$ parsecs) are likely to dominate the pulsar contribution to the positron spectrum, especially energies above $\sim 50 \mathrm{GeV}$. More distant pulsars, however, are still anticipated to play an important role in the lower energy range of the PAMELA positive excess. In Fig. 4, we show a combination of pulsar contributions to the high energy positron spectrum and the positron fraction. In particular, we include the contribution from all pulsars more distant than 500 parsecs (using a rate of 4 pulsars per century, as shown in the lower frames of Fig. 1) and the contributions from B0656+14 and Geminga (using $3 \times 10^{47} \mathrm{erg}$ in electron-positron pairs from each and a spectral index of 1.5). As can be seen in the right frame, such a combination can provide a good fit to the preliminary measurements of PAMELA and accommodates for a rising positron fraction even beyond $\sim 100 \mathrm{GeV}$.

A comment regarding this result is in order. In describing the contribution from all pulsars throughout the Milky Way, we have adopted a spectrum with an index of 1.6 and an exponential cutoff above $80 \mathrm{GeV}$. In contrast, we have used a higher cutoff $(600 \mathrm{GeV})$ and slightly harder slope (1.5) for the nearby B0656+14 and Geminga pulsars. These results do not contradict each other, for the average injected spectrum from Ref. [18] results from the sum over a realization with wide variability of pulsar properties, including injected energy and spectral index. In this respect, the results presented here are only demonstrative of the fact that a significant role of nearby pulsars, while not required to explain present data, is consistent with them, in which case they should dominate the high energy tail. If this is the case, interesting observational signatures are possible, one of which is discussed in the next section. In no case the 

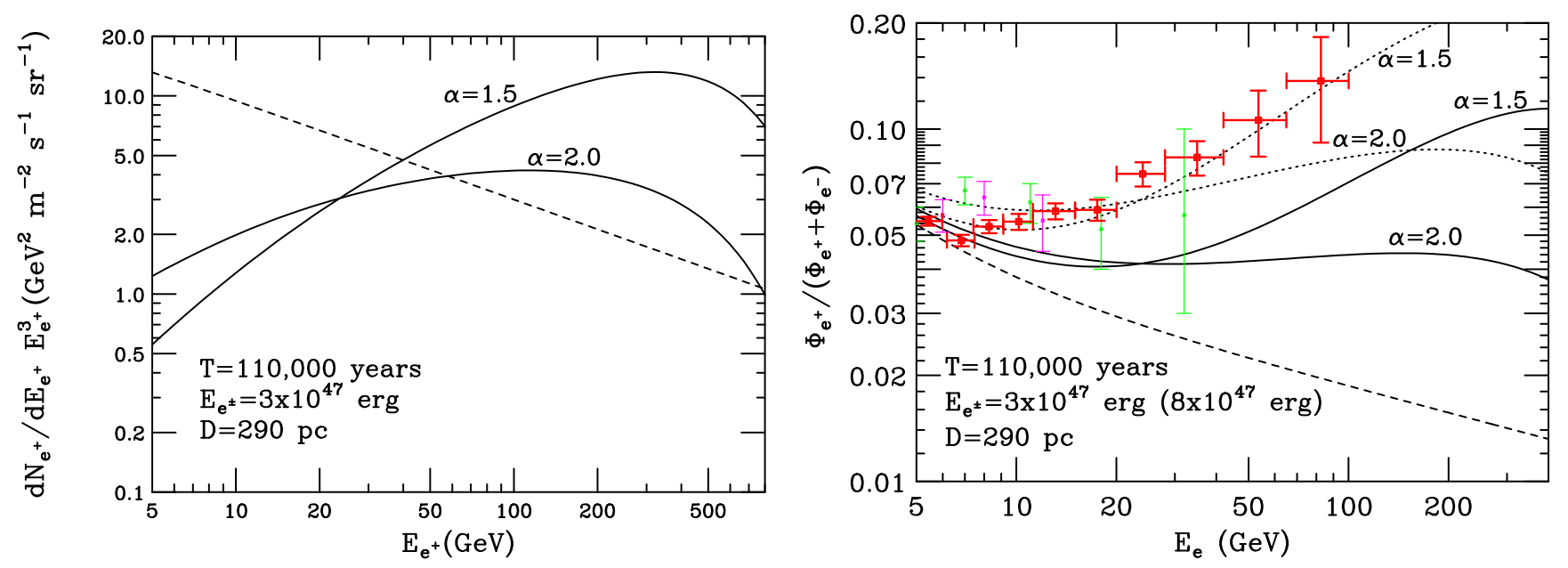

FIG. 3: As in Fig. 2. but from the nearby pulsar B0656+14. The solid lines correspond to an energy in pairs given by $3 \times 10^{47}$ erg, while the dotted lines require an output of $8 \times 10^{47} \mathrm{erg}$.
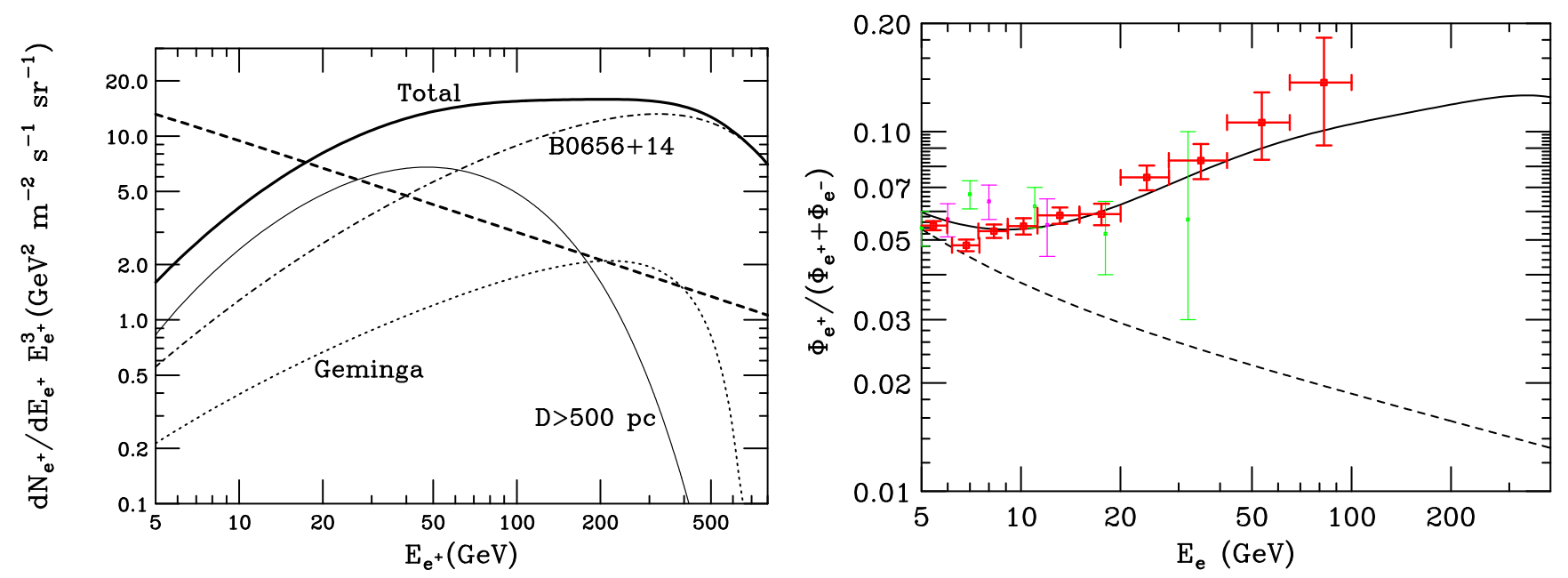

FIG. 4: The positron spectrum and positron fraction from the sum of contributions from B0656+14, Geminga, and all pulsars farther than 500 parsecs from the Solar System.

high-energy spectra presented here should be considered as a robust prediction, since they depend crucially on the detailed spectral properties of B0656+14, Geminga or and other nearby, mature pulsars that contribute significantly to the high energy positron spectrum.

\section{DISTINGUISHING BETWEEN PULSAR AND DARK MATTER ORIGINS OF HIGH ENERGY COSMIC RAY POSITRONS}

The positron fraction reported by PAMELA taken alone is likely insufficient to distinguish between dark matter and pulsar origins of this signal. In this section we discuss an additional measurement which may help to resolve this issue. In particular, even after the diffusive propagation of electrons and positrons from pulsars is taken into account, at sufficiently high energies a small dipole anisotropy should be present in the direction of the dominant nearby source(s). In a very general way, the anisotropy associated with diffusive propagation can be written as:

$$
\delta=\frac{I_{\max }-I_{\min }}{I_{\max }+I_{\min }}=\frac{3 K\left|\nabla\left(d N_{e} / d E_{e}\right)\right|}{c\left(d N_{e} / d E_{e}\right)},
$$

where $\nabla\left(d N_{e} / d E_{e}\right)$ is the gradient of the electron/positron density. The measurement of such an anisotropy in a statistical significant manner requires a large number of electron/positron events. For example, in order to detect an 


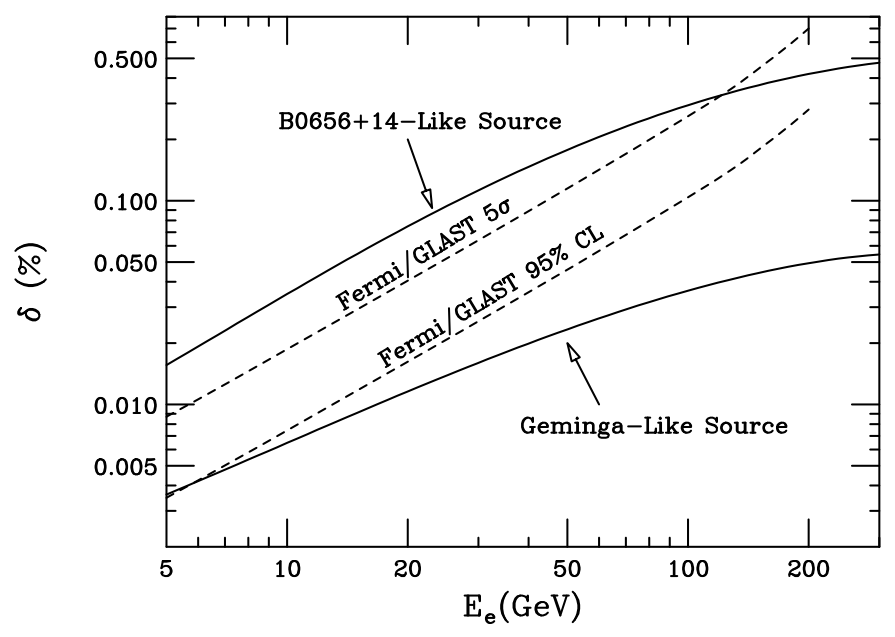

FIG. 5: The dipole anisotropy in the electron+positron spectrum from a source 110,000 years old at a distance of 290 pc (B0656+14-like) and from a source 370,000 years old at a distance of 157 pc (Geminga-like). In each case, we have normalized the energy output to match the PAMELA data and have used a spectral shape of $d N_{e} / d E_{e} \propto E_{e}^{-1.5} \exp \left(-E_{e} / 600 \mathrm{GeV}\right)$. Also shown as dashed lines is the sensitivity of the Fermi gamma-ray space telescope to such an anisotropy (after five years of observation). The Fermi sensitivity shown is for the spectrum integrated above a given energy.

anisotropy at the $2 \sigma$ level, one needs to fulfill the condition $\delta \gtrsim 2 \sqrt{2}\left(\dot{N}_{\text {ev }} t_{\text {obs }}\right)^{-1 / 2}$, where $\dot{N}_{\text {ev }}$ is the rate of events detected per unit time above a given threshold and $t_{\mathrm{obs}}$ is the observation time.

In addition to studying the gamma-ray sky, the Fermi gamma-ray telescope will also be able to measure a flux of electrons (and positrons, though without charge discrimination) at a rate of approximately $3 \times 10^{7}$ electrons per year above $10 \mathrm{GeV}$ [30]. This implies that Fermi should be able to detect (at the $2 \sigma$ confidence level) a dipole anisotropy in the electron flux above $10 \mathrm{GeV}$ if $\delta \gtrsim 0.05 \%$ in one year or $\delta \gtrsim 0.03 \%$ in 5 years $^{1}$.

In Fig. 5 we plot the level of anisotropy expected for a Geminga-like and a B0656+14-like pulsar if they are responsible for the majority of the observed positron excess. The two dashed lines show the sensitivities of Fermi to anisotropy at $95 \%$ confidence level and at $5 \sigma$ confidence level, after five years of observation (integrated above a given energy). We find that Fermi should be capable of identifying a single local source (or multiple sources in the same direction of the sky) if that source injected the bulk of its electrons/positrons within the last few hundred thousand years (the B0656+14-like and Geminga-like cases correspond to injection 110,000 and 370,000 years ago, respectively). If only a fraction of the high energy positrons observed by PAMELA originate from a given nearby pulsar, the corresponding solid lines shown in Fig. 5 should be multiplied (reduced) by this factor. Also note that B0656+14 and Geminga lie in similar directions in the sky, so they are expected to contribute the same overall dipole anisotropy.

Alternatively, if dark matter annihilations throughout the Milky Way's halo are primarily responsible for the excess in the high energy cosmic ray positron spectrum, a small dipole anisotropy in the direction of the Galactic Center could also be generated. Fortunately, both B0656+14 and Geminga are in approximately the opposite direction, allowing for a potentially unambiguous discrimination between these possibilities. In the special and relatively unlikely case that a nearby dark matter subhalo in the direction of B0656+14/Geminga is responsible for the observed flux, it would be difficult to distinguish between pulsar and dark matter origins using this technique.

If anisotropy studies should prove inconclusive in resolving this issue, other information could be inferred from the shape of the positron fraction and of the overall electron/positron spectrum. Peculiar shapes can result from the superposition of the overall pulsar spectrum plus local contributions (see, for example, Fig. 4 or Ref. [16]). Future studies of the electron and positron spectra at higher energies will be especially important, as the spectral cutoff in the pulsar case is typically expected to be smoother and less sudden than that predicted from annihilating dark matter. Furthermore, combining electron/positron measurements with those of antiprotons, antideuterons and diffuse gammarays may prove useful in distinguishing between these possibilities. Population studies of pulsars in the gamma-ray

\footnotetext{
${ }^{1}$ Note that, extrapolating from the data reported in [2], PAMELA can collect $\sim 10^{5}$ useful electrons plus positrons events per year above $1.5 \mathrm{GeV}$. For the range of interest here this is several orders of magnitude below Fermi and unlikely to lead to a meaningful constraint on the anisotropy of high energy charged leptons.
} 
band by Fermi are also expected to refine theoretical predictions and shed light on this issue.

\section{CONCLUSIONS}

The results recently reported by PAMELA strongly indicate the existence of a primary source or sources of high energy cosmic ray positrons. This result is unexpected and very interesting, even if of purely astrophysical origin. Several papers have appeared recently which discuss this signal within the context of dark matter annihilations. In this article, we have instead explored the possibility that the observed flux of high energy positrons is the result of electron-positron pairs being produced in nearby and galactic pulsars. We find that pulsars throughout the Milky Way, and a small number of nearby mature pulsars, such as B0656+14 and Geminga, could each plausibly generate the observed flux of positrons. To normalize the overall flux, on the order of a few percent of the pulsars' spin down power is required to be transferred into the production of electron-positron pairs. The prediction in the case of the sum of all pulsars in the Galaxy appears somewhat more robust in that it relies on the average statistical properties of these astrophysical objects rather than on the specific characteristics of nearby pulsars. It is remarkable that in this case, reasonable values for the parameters can lead to a positron spectrum consistent with the observations. Also, a pulsar origin would naturally fit the absence of an excess in the anti-proton data [31], since differently from dark matter scenarios no hadronic cascades are associated with the production of pairs in the magnetospheres.

If a single or small number of nearby pulsars dominate the high energy positron spectrum, an observable dipole anisotropy in the electron-positron spectrum may be present. In particular, we find that the Fermi gamma-ray space telescope would be able to detect the anisotropy generated by a few hundred thousand year old or younger source with greater than $2 \sigma$ significance. A 100,000 year old source, such as B0656+14, would produce an anisotropy that could be detected by Fermi with greater than $5 \sigma$ significance. This provides a valuable test for distinguishing between pulsar and dark matter origins of the observed cosmic ray positron spectrum, complementary to the spectral shape and additional information from antiprotons and gamma-rays.

\section{Acknowledgments}

DH is supported by the Fermi Research Alliance, LLC under Contract No. DE-AC02-07CH11359 with the US Department of Energy and by NASA grant NNX08AH34G.

[1] http://pamela.roma2.infn.it/index.php

[2] O. Adriani et al., arXiv:0810.4995 [astro-ph].

[3] S. W. Barwick et al. [HEAT Collaboration], Astrophys. J. 482, L191 (1997) arXiv:astro-ph/9703192; S. Coutu et al. [HEAT-pbar Collaboration], in Proceedings of 27th ICRC (2001).

[4] M. Aguilar et al. [AMS-01 Collaboration], Phys. Lett. B 646, 145 (2007) arXiv:astro-ph/0703154.

[5] L. Bergstrom, T. Bringmann and J. Edsjo, arXiv:0808.3725 [astro-ph].

[6] M. Cirelli and A. Strumia, arXiv:0808.3867 [astro-ph].

[7] V. Barger, W. Y. Keung, D. Marfatia and G. Shaughnessy, arXiv:0809.0162 [hep-ph].

[8] I. Cholis, L. Goodenough, D. Hooper, M. Simet and N. Weiner, arXiv:0809.1683 [hep-ph].

[9] M. Cirelli, M. Kadastik, M. Raidal and A. Strumia, arXiv:0809.2409 [hep-ph].

[10] N. Arkani-Hamed, D. P. Finkbeiner, T. Slatyer and N. Weiner, arXiv:0810.0713 [hep-ph].

[11] J. Hisano, S. Matsumoto, M. M. Nojiri and O. Saito, Phys. Rev. D 71, 063528 (2005) [arXiv:hep-ph/0412403] S. Profumo, Phys. Rev. D 72, 103521 (2005) arXiv:astro-ph/0508628]; M. Cirelli, A. Strumia and M. Tamburini, Nucl. Phys. B 787, 152 (2007) arXiv:0706.4071 [hep-ph]]; J. March-Russell, S. M. West, D. Cumberbatch and D. Hooper, JHEP 0807, 058 (2008) arXiv:0801.3440 [hep-ph]].

[12] P. Grajek, G. Kane, D. J. Phalen, A. Pierce and S. Watson, arXiv:0807.1508 [hep-ph].

[13] D. Hooper, J. E. Taylor and J. Silk, Phys. Rev. D 69, 103509 (2004) arXiv:hep-ph/0312076.

[14] E. A. Baltz et al., JCAP 0807, 013 (2008) arXiv:0806.2911 [astro-ph]].

[15] D. Hooper, Phys. Rev. D 77, 123523 (2008) arXiv:0801.4378 [hep-ph]]; E. Borriello, A. Cuoco and G. Miele, arXiv:0809.2990 [astro-ph].

[16] F. A Aharonian, A. M. Atoyan and H. J. Volk, Astron. Astrophys. 294, L41-L44 (1995).

[17] C. Grimani, Astron. Astrophys. 474, 339 (2007).

[18] L. Zhang and K. S. Cheng, Astron. Astrophys. 368, 1063-1070 (2001).

[19] I. Buesching, O. C. de Jager, M. S. Potgieter and C. Venter, arXiv:0804.0220 [astro-ph].

[20] http://fermi.gsfc.nasa.gov/ 
[21] M. A. Ruderman and P. G. Sutherland, Astrophys. J. 196, 51 (1975).

[22] J. Arons, Astrophys. J. 266, 215A (1983).

[23] K.S. Cheng, C. Ho, M. Ruderman, Astrophys. J. 300, 522C (1986); Astrophys. J. 300, 500C (1986).

[24] M.S. Longair, "High Energy Astrophysics", Vol. II Cambridge University Press; 2nd edition (1994). S.L. Shapiro S.A. Teukolsky ,"Black Holes, White Dwarfs and Neutron Stars: The Physics of Compact Objects", Wiley-Interscience (1983). M. Vietri, "Foundations of High-Energy Astrophysics", University Of Chicago Press (2008).

[25] X. Chi, K. S. Young, and E. C. M. Young, Astrophys. J. 459, L83 (1996).

[26] B. Paczynski, Astrophys. J. 348, 485 (1990); S. J. Sturner and C. D. Dermer, Astrophys. J. 461, 461 (1996).

[27] I. V. Moskalenko and A. W. Strong, Astrophys. J. 493, 694 (1998) arXiv:astro-ph/9710124.

[28] G. G. Raffelt, arXiv:astro-ph/0701677.

[29] C. Amsler et al. [Particle Data Group], Phys. Lett. B 667 (2008) 1.

[30] A. A. Moiseev, J. F. Ormes and I. V. Moskalenko, arXiv:0706.0882 [astro-ph].

[31] O. Adriani et al., arXiv:0810.4994 [astro-ph]. 